\title{
Urban vegetation change after a hundred years in a tropical city (San José de Costa Rica)
}

\author{
Julián Monge-Nájera \& Gabriela Pérez-Gómez \\ Laboratorio de Ecología Urbana, Vicerrectoría de Investigación, Universidad Estatal a Distancia, 474-2050 San Pedro \\ de Montes de Oca, San José, Costa Rica; julianmonge@gmail.com, gabytta1985@gmail.com
}

Received 01-VI-2010. C Corrected 28-VII-2010. Accepted 06-VIII-2010.

\begin{abstract}
Urban vegetation is of key importance because a large proportion of the human population lives in cities. Nevertheless, urban vegetation is understudied outside central Europe and particularly, little is known about the flora of tropical Asian, African and Latin American cities. We present an estimate of how the vegetation has changed in the city of San José, Costa Rica, after about one century, with the repeat photography technique (based on a collection of $19^{\text {th }}$ and early $20^{\text {th }}$ century photographs by José Fidel Tristán and others) and with data from the Costa Rican National Herbarium. We found little vegetation change in the landscape of San José during the 20th century, where a total of 95 families and 458 species were collected in the late 19th and early 20th century. The families with most species were Asteraceae, Fabaceae, Poaceae, Lamiaceae, Euphorbiaceae, Solanaceae, Cyperaceae, Acanthaceae, Malvaceae, Piperaceae and Verbenaceae. Similar results have been found in Europe, where the number of plant species often is stable for long periods even when the individual species vary. Rev. Biol. Trop. 58 (4): 1367-1386. Epub 2010 December 01.
\end{abstract}

Key words: Urban flora, effects of urbanization, city landscape, photographic comparison, species list.

Urban vegetation has mostly been studied in central Europe, where about $50 \%$ of species are alien, half of them introduced before the $15^{\text {th }}$ century. Despite the heavy traffic among European cities, five centuries have not been enough to homogenize their flora: the communities of species introduced after the year 1500 are characteristic of each city (Frank et al. 2008).

For animals, which particular species occur in cities is predicted by the "environmental filtering model" that in turn is based on plants. The model states that (1) there is natural selection of species living in urban ecosystems, (2) plants define key habitat characteristics and (3) habitats define which animals can live in the city (Williams et al. 2009). Generally, moderate urbanization produces some increase in plant biodiversity but is deleterious for invertebrates and mammals. A high level of urbanization is correlated with fewer species of plants, invertebrates, amphibians, reptiles, birds and mammals, possibly because humans willingly introduce plant species, but not animals, to their gardens (McKinney 2008).

Outside central Europe, urban vegetation is understudied but there are some recent data from Plymouth, England, where alien species increase with urbanization (Kent et al. 2001). Also in England, gardens in Sheffield have a total of 1166 plant species (70\% alien) and twice the garden size means $25 \%$ more species. In these gardens there are $63 \%$ biennials/perennials, $18 \%$ shrubs, $10 \%$ annuals and $8 \%$ trees (Smith et al. 2006).

In Anglosaxon and French North America, there is a surprising scarcity of recent studies on urban biotas, but some work has been done. 
In the city of Halifax (Nova Scotia, Canada), soil moisture and light determine which species are present. Taxa adapted naturally to rock, grassland and flooded habitat find an analog habitat in the city and thrive (Lundholm \& Marlin 2006).

The New York metropolitan region has 556 woody species and non-native invasive species are becoming more common (Clemants \& Moore 2004). In the Pelham Bay Park, New York City, native species went from $72 \%$ to $60 \%$ and $26 \%$ of natives disappeared in 50 years (especially herbaceous and meadow-type plants, DeCandido 2004).

Even though recent studies are scarce, a meta-analysis found 79 studies of species richness with geographic data for New York City; of these, 17 studies found a decrease in species richness, six an increase and three found no change. However, all studies reported an increasing number of exotic species (Puth \& Burns 2008).

Tropical cities are in areas of high biodiversity but little is known about Asian and especially African cities regarding urban flora. In Latin America the situation is better but worldwide no floral lists exist for the 50 most populated cities (Clemants 2002). In Jinan City, China, a methodological comparison found that gradient analysis from the urban center to the fringe gives better estimates of the urban flora than the traditional block-area analysis (Kong \& Nakagoshi 2005). In Taipei, green areas have 164 tree species (few shared among sites) and large evergreen native species dominate. Larger parks have higher richness, more landscape fidelity to the original vegetation, and more rare and endemic species ( $\mathrm{Jim} \&$ Chen 2007). In Africa, the urban areas of the Nile Delta (Egypt) have vegetation that is mainly correlated with moisture, $\mathrm{pH}$, fertility and texture gradients, but plants always occupy sites similar to their natural habitats (Shaltout \& El-Sheikh 2002).

Latin America has a long history of scientific study of urban biota, particularly the plants and there are several recent studies from México, Peru, Brazil, Chile and Argentina.
In the city of Ensenada, Mexico, there are 161 species, 61\% non-native (Garcillán et al. 2009). In Mexico City, trees are stressed from dry wind and unfavorable water flow caused by the pavement (Barradas 2000).

Brazil has the largest urban forest in world (Tijuca: 3300 hectares) but it is being stressed by roads because roads are surrounded by invasive species that burn easily. The fires in turn open adjacent areas to more invasive vegetation and the damage spreads (Matos et al. 2002). There are very few studies of plants that grow on walls but in Jundiai, Brazilian, walls have a biodiversity of 28 species (dos Reis et al. 2006).

Normally, satellites are not used to study urban vegetation but in Arequipa, Peru, satellite images show that desert vegetation is being lost because of urban expansion (Polk et al. 2005). However, only ground work can reliably identify species and this kind of work has shown that temperate South America is not different from North America and Europe: at least half of the plant species in the Argentinean cities of Mendoza and Rosario are introduced. In Luján de Cuyo, Mendoza, 61 species were identified: 69\% introduced (Méndez 2005). The vacant lots of Rosario each have one dominant species, a few abundant species and many rare species. Therophytes predominate and the proportions of indigenous and introduced species are similar (Franceschi 1996).

Chile is the Latin American country with the largest number of recent studies. Synanthropic communities in an urban footpath of Valdivia represents six associations and two communities (Finot \& Ramírez 1998). In Concepción, green areas are dominated by nonnative ornamental species (Paucharda et al. 2006). The distribution of urban vegetation reflects social inequalities. In Santiago, poor areas can have ten times less plant cover than rich neighbourhoods (Hernández 2008), similar to other countries (Pedlowski et al. 2002). However, workshops in poor areas of cities can result in an improvement of their vegetation (Garzón et al. 2004). 
In Costa Rica, there is a long history of study of urban plants that began with the National Museum's collection efforts in the late $19^{\text {th }}$ century, but little has been published. Méndez \& Fournier (1980) and Monge-Nájera et al. $(2002 \mathrm{a}, \mathrm{b})$ studied the lichens and their relationship with air pollution. The use of European lichens proved succesful when they were transplanted to this Tropical city (Grüninger \& Monge-Nájera 1988). Francisco Fallas made checklists and abundance estimates of urban herbs in the late 1970's but to our knowledge he did not publish them. The program "Costa Rica: Jardín Botánico de América Tropical” produces manuals and labels for urban vegetation (www.hjimenez.org) and there is a program to provide urban parks with butterflies and their host plants (http://www.lrsarts.com/ plas/index.html).

The biodiversity in patches of urban vegetation can be surprinsingly high, at least in Costa Rica. For example, after 50 years, in only one hectare of urban vegetation in San José, there are 432 plant species (Di Stéfano et al. 1995, Nishida et al. 2009), a full new lichen family with a novel symbiotic lifestyle (Eremithallus costaricensis; Lücking et al. 2008), 200 butterfly species (Nishida et al. 2009) and a new species of Hymenoptera, Meteorus oviedo (Shaw \& Nishida 2005).

We present an estimate of how the vegetation has changed in the city of San José, Costa Rica, after about one century, with a technique called repeat photography. We do not know of repeat photography studies on urban Costa Rican vegetation of San José, but the technique was used in Costa Rica by Horn (1989) to assess changes in the páramo habitat.

\section{MATERIALS AND METHODS}

We used a collection of photographs taken in the late $19^{\text {th }}$ and early $20^{\text {th }}$ centuries from the José Fidel Tristán Fernández Collection in the Archivo Nacional de Costa Rica and others reproduced by Leiva (2004). The sites were re-photographed on December 11, 2008 with a Nikon Coolpix 8800 camera (8 megapixels;
Fig. 1). The repeat photography technique, developed in 1880 (Webb et al. 2010) is good for detailed analysis (Hendrick \& Copenheaver 2009) and is cost- effective (Robert et al. 2010). We used digital repeat photography, which is fast, detailed and reliable; can be stored for future corroboration and comparison; includes rich data that may become useful in the future; and can classify and measure information automatically (Crimmins \& Crimmins 2008).

We re-photographed nine sites (exact year of original photograph included when known): Site 1 Catedral Metropolitana Street: 0 Avenues: 2-4 (1896); Site 2 Catedral Metropolitana St. 0 Av. 0-2 (c. 1910); Site 3 Kiosco Parque Morazán St. 5-7 Av. 3 (1914); Site 4 Colegio de Señoritas St. 3-5 Av. 4-6 (1914); Site 5 Paseo Colón St. 36 Av. 0, looking east (1899); Site 6 Paseo Colón St. 36 Av. 0 looking west; Site 7 Antigua Casa Presidencial St. 15 Av. 7; Site 8 Estación del Ferrocarril al Atlántico St. 21-23 Av. 3; Site 9 Hospital de Niños St. 20 Av. 0.

We calculated \% cover by clipping and weighing sections from photographs printed on standard bond paper; for example, if the clippings from buildings represented $20 \%$ of the total weight of the photograph, we recorded that buildings represented $20 \%$ of the image. This technique is compared with others by Monge Nájera et al. (2002b).

We also present an analysis of plants collected in cantón de San José from 1885 through 1945 by the Museo Nacional staff (Base de Datos, Herbario Nacional, updated to April 13, 2009).

\section{RESULTS}

After about a century, the main change in San José city photographs is the much larger number of people. The reduction in vegetation affects grasses, shrubs and trees, but is small; the increase in buildings, streets, vehicles, sidewalks, lamps and signs is also small (Fig. 2).

A total of 95 families and 458 species were collected in the late 19th and early 20th century. 

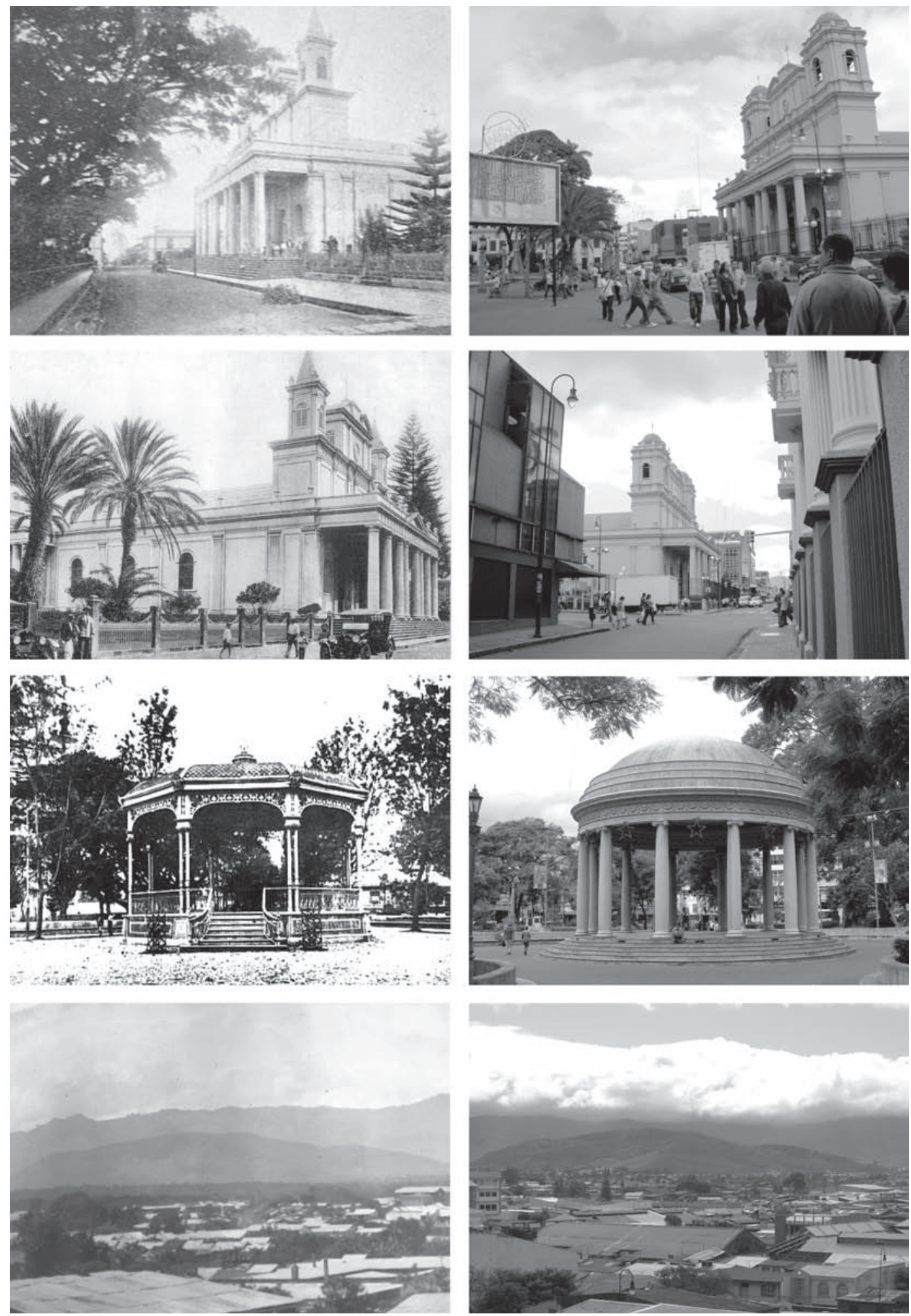

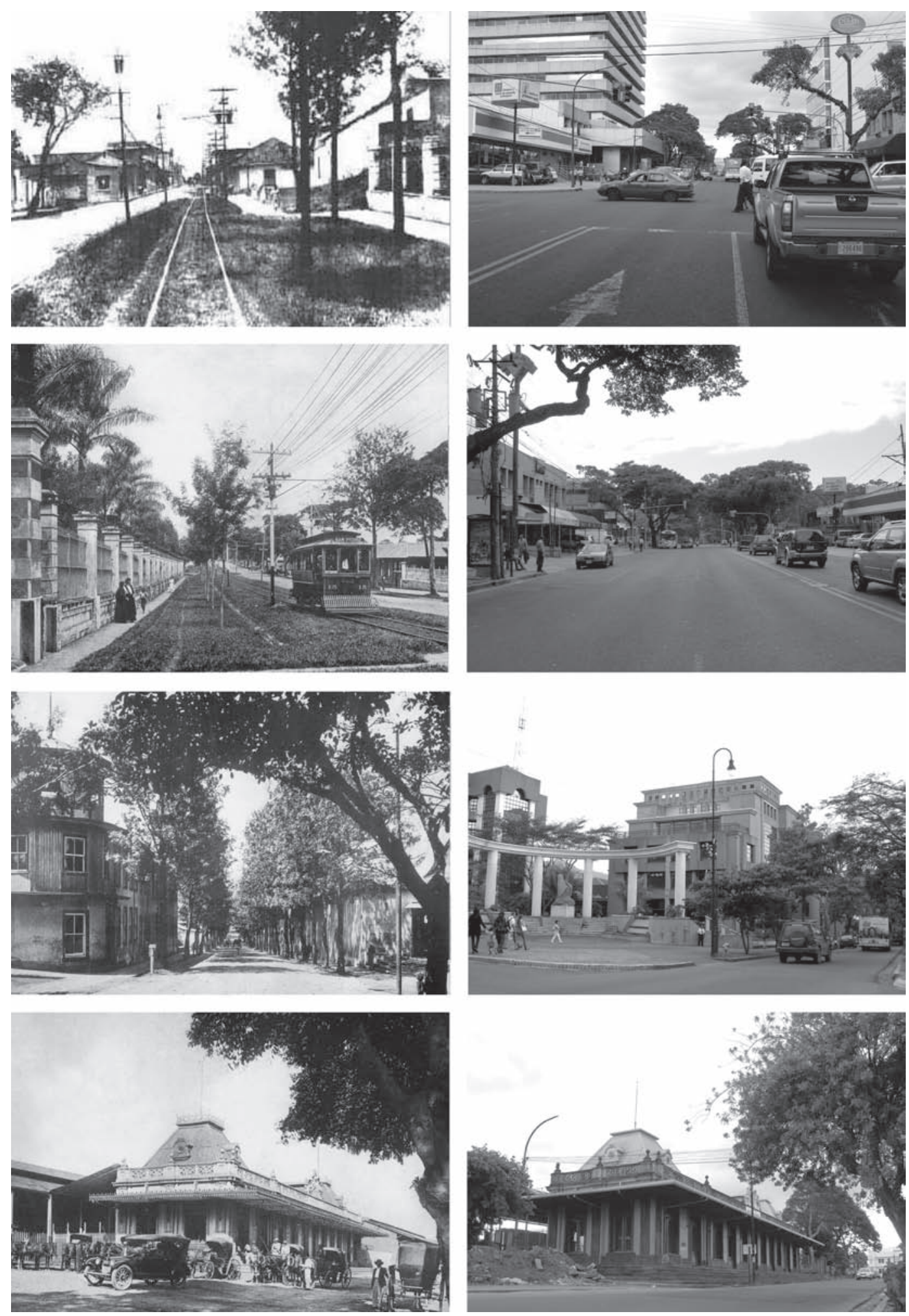

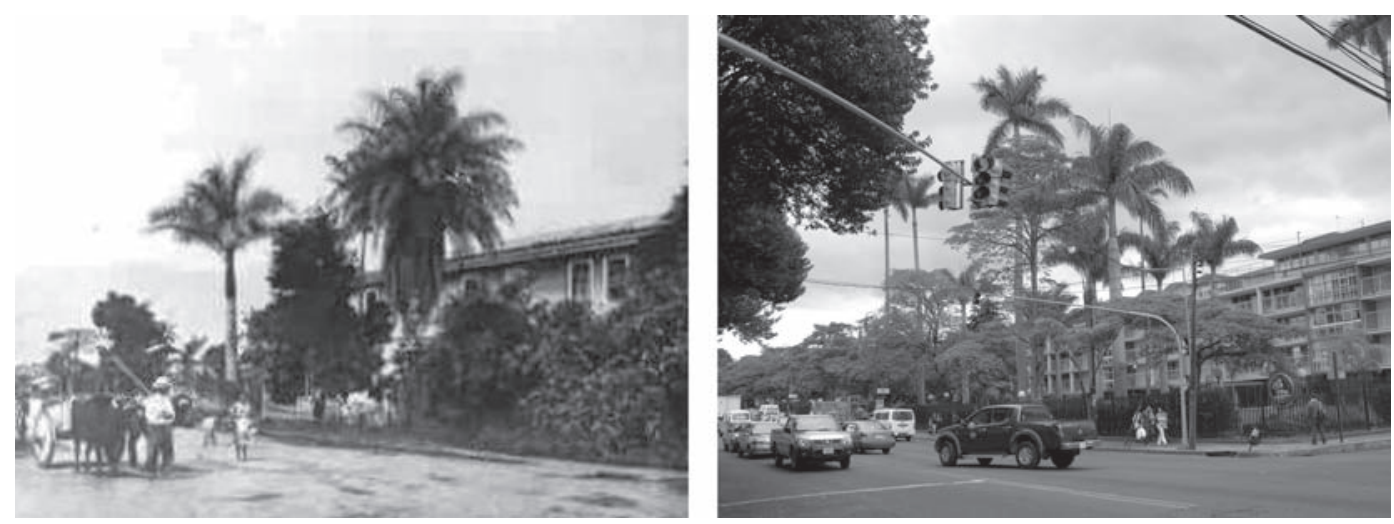

Fig. 1. Images used for the comparison: City of San José, Costa Rica.

NOTE: The original images used in the measurements appear in the Internet edition.

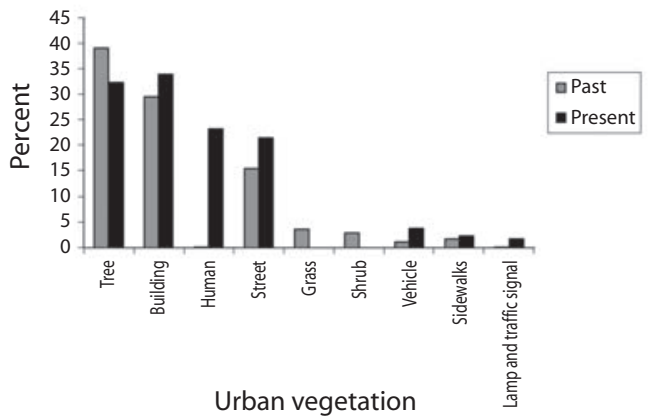

Fig. 2. Change in vegetation, infrastructure, vehicles and humans in photographs from the city of San José, Costa Rica, from a century ago to the present $(\%$ cover in photographs).

The families with most species were Asteraceae, Fabaceae, Poaceae, Lamiaceae, Euphorbiaceae, Solanaceae, Cyperaceae, Acanthaceae, Malvaceae, Piperaceae and Verbenaceae (Appendix 1).

\section{DISCUSSION}

Repeat photography was used in Costa Rica by Horn (1989) to assess changes in the paramo habitat (she found very little change) but we were unable to find a similar study of urban vegetation. Nevertheless, studies about plant biodiversity after long periods suggest that our results are not unusual. For example, after 50 years, Brussels has the same total number of plant species that it had in 1940, albeit the individual species change and the same applies to other areas in much longer time spans (Chocholouškováa \& Pyšek 2003). We cannot make a comparison of species from circa 1900 to circa 2000 in San José because urban vegetation has rarely been collected in recent decades.

The increase in human presence in the city landscape is explained by the population growth of downtown San José or Cantón Central (from about 30000 when the first photographs used in this study were taken circa 1900 to 356000 when the sites were rephotographed in 2009; see: Centro Centroamericano de Población and Instituto Nacional de Estadística y Censos 2002). The small increase in the number of vehicles is an underestimation: the 2008 photographs were purposefully taken in low traffic periods to obtain a better view of the scenes.

Central European cities have a mean of 646 plant species/city and larger cities have more species (Pysek 1998), thus, considering that San José was and is a small city, the total of 458 species recorded is within the expected range.

Successful urban plants tend to belong to species adapted to natural habitats with strong sunlight, abundant nitrogen and low 
water levels (Shaltout \& El-Sheikh 2002, Chocholouškováa \& Pyšek 2003, Lundholm \& Marlin 2006), so the presence of many Asteraceae, Fabaceae, Poaceae, Lamiaceae, Euphorbiaceae, Solanaceae, Cyperaceae, Acanthaceae, Malvaceae, Piperaceae and Verbenaceae is not surprising. Many of the plant species in Costa Rica are introduced (Chacón \& Saborío 2005) and here again the situation is similar to that in other countries.

Urban trees mitigate global warming (Abdollahi et al. 2000, McPherson et al. 2008), significantly reduce urban heat islands (Huang et al. 2009) and can sequester about $100 \mathrm{~kg}$ of air pollutants per hectare of urban forest (Vilela-Lozano 2004). Furthermore, urban vegetation protects many species in the five kingdoms (Dana et al. 2002, Smith et al. 2006), including valuable rare species (Maurer et al. 2000, Williams et al. 2009). For these and other reasons, the study and management of urban vegetation is of great importance.

Genetic diversity is low in urban plants and they are less prepared to cope with environmental change (Knapp et al. 2009), so periodic monitoring is needed to conserve original species as well as any others in need of protection (Godefroid 2001). Citizens can learn to effectively take advantage of urban vegetation (Garzón et al. 2004) and to recognize historical changes in the city scene (e.g. the repeat photography groups in www.flickr. com), not only for cultural reasons, but also to influence the administration of the urban flora by local governments.

Future studies of urban vegetation in San José could investigate these hypotheses:

- Floristic composition results from the interaction of human density, water availability, temperature, altitude and soil (Dana et al. 2002, Chocholouškováa \& Pyšek 2003, Fanelli \& Tescarollo 2006, Altobelli et al. 2007).

- Ecological succession starts with ruderal annual plants, followed by perennials (Prach et al. 2001).

- Alien species benefit more from human activity (Niggermann 2009).
- With global warming, species adapted to colder climate will become less common and vice versa.

\section{ACKNOWLEDGMENTS}

We thank Hubert Blanco (Archivos Nacionales), Colegio Superior de Señoritas, María José Guerra Araus (Herbario Nacional), Sergio Aguilar, Sergio Quesada, Karla Vega, Andrea Sánchez and María Acuña for their assistance. We specially thank Sally P. Horn (University of Tennessee, Knoxville) for suggestions to improve the manuscript and for advice on repeat photography.

\section{RESUMEN}

La vegetación urbana es de vital importancia ya que una proporción importante de la población humana vive en ciudades. Sin embargo, esta vegetación es poco estudiada fuera del centro de Europa y se sabe particularmente poco sobre la flora urbana de las ciudades tropicales de Asia, África y América Latina. Aquí presentamos una estimación de cómo ha cambiado la vegetación en la ciudad de San José, Costa Rica, durante un siglo, con la técnica de la fotografía repetida (sobre la base de una colección de fotografías del siglo XIX y principios del siglo XX hechas por José Fidel Tristán y otros) y con los datos del Herbario Nacional de Costa Rica. Encontramos pocos cambios en el paisaje de San José durante el siglo XX. En la ciudad se recolectaron 95 familias y 458 especies entre finales del siglo XIX y principios del XX. Las familias con más especies fueron Asteraceae, Fabaceae, Poaceae, Lamiaceae, Euphorbiaceae, Solanaceae, Cyperaceae, Acanthaceae, Malvaceae, Piperaceae y Verbenaceae. Los resultados son similares a los de Europa, donde el número de especies de plantas a menudo es estable durante largos períodos, aunque las especies individuales varíen.

Palabras clave: Flora urbana, efectos de la urbanización, paisaje de ciudad, comparación fotográfica, lista de especies.

\section{REFERENCES}

Abdollahi, K., Z. Ning \& A. Appeaning. 2000. Global climate change and the urban forest. Franklin, California, USA.

Altobelli, A., E. Bressan, E. Feoli, P. Ganis \& F. Martini. 2007. Improving knowledge of urban vegetation by 
applying GIS technology to existing databases. Appl. Veget. Sci. 10: 203-210.

Aparecida dos Reis, V., J.A Lombardi \& R.A. Figueiredo. 2006. Diversity of vascular plants growing on walls of a Brazilian city. Urban Ecosyst. 9: 39-43.

Barradas, V. 2000. Energy balance and transpiration in an urban tree hedgerow in Mexico City. Urban Ecosyst. 4: 55-67.

Chacón, E. \& G. Saborío. 2005. Sistema de Información de las Especies Invasoras en Costa Rica. Propuesta ante la OEA. Organización de Estados Americano (OEA), Washington, D.C. (www.oas.org/dsd/IABIN/Component2/CostaRica. Downloaded June 1, 2010).

Chocholoušková, Z. \& P. Pyšek. 2003. Changes in composition and structure of urban flora over 120 years: a case study of the city of Plzeň. Flora- Morphology, Distribution. Functional Ecol. Plants 198: 366-376.

Centro Centroamericano de Población \& Instituto Nacional de Estadística y Censos. 2002. Costa Rica: estimaciones y proyecciones de población 1970-2100 actualizadas al año 2000 y evaluación del Censo 2000 y otras fuentes de información. Centro Centroamericano de Población \& Instituto Nacional de Estadística y Censos, San José, Costa Rica.

Clemants, S. 2002. A Short Bibliography of Urban Floras. Urban habitats 1: 1541-7115.

Clemants, S. \& G. Moore 2004. The Changing Flora of the New York Metropolitan Region. Urban Habitats 3: $192-210$

Crimmins, M. \& T. Crimmins. 2008. Monitoring Plant Phenology Using Digital Repeat Photography. Environ. Manage. 41: 1432-1009.

Dana, E.D, S. Vivas \& J.F. Mota. 2002. Urban vegetation of Almería City - a contribution to urban ecology in Spain. Landscape Urban Plann: 203-216.

De Candido, R. 2004. Recent changes in plan species diversity in urban Pelham Bay Park, 1997-1998. Biol. Conserv. 120: 129-136.

Di Stéfano, J.F., V. Nielsen, J. Hoomans \& L.A. Fournier. 1995. Regeneración de la vegetación arbórea en una pequeña reserva forestal urbana del premontano húmedo, Costa Rica. Rev. Biol. Trop. 41:7-21.

Fanelli,P.\& A.Tescarollo. 2006. Ecological indicator applied to urban and suburban floras. Ecol. Indic. 6: 444-457.

Finot, V. \& C. Ramírez. 1998. Fitosociología de la vegetación ruderal de la ciudad de Valdivia (X RegiónChile). Studia Bot. 17: 69-86.
Franceschi, E.A. 1996. The ruderal vegetation of Rosario City, Argentina. Landscape Urban Plann. 34: 11-18.

Frank, A., M. La Sorte, P. McKinney, S. Klotz, G. Rapson, L. Celesti-Grapow \& K. Thompson. 2008. Distance decay of similarity among European urban floras: the impact of anthropogenic activities on Biodiversity. Global Ecol. Biogeogr. 17: 363-371.

Garcillán, P.P., J. Rebman \& F. Casillas. 2008. Analysis of the non-native flora of Ensenada, a fast growing city in north western Baja California. Urban Ecosyst. 12: 449-463.

Garzón, B., N. Brañes, M. Albés \& A. Anuad. 2004. Vegetación tropical y habitat popular: el caso de San Miguel de Tucumán. Bol. Inst. Vivienda 18: 21-42.

Godefroid, S. 2001. Temporal analysis of the Brussels flora as indicator for changing environmental quality. Landscape Urban Plann. 52: 203-224.

Grüninger, W. \& J. Monge-Nájera. 1988. Use of the temperate lichen Hypogymnia physodes (Parmeliaceae) to valuate air pollution in the Tropics. Rev. Biol. Trop. 36: 545-547.

Hendrick , L. \& C. Copenheaver. 2009. Using Repeat Landscape Photography to Assess Vegetation Changes in Rural Communities of the Southern Appalachian Mountains in Virginia, USA. Mountain Res. Devel. 29: 21-29.

Hernández P.H.J. 2008. La situación del arbolado urbano en Santiago. Revista de Urbanismo (Chile) (http://revistaurbanismo.uchile.cl. Downloaded June 7, 2010).

Horn, S. 1989. The Inter-American highway and human disturbance of páramo vegetation in Costa Rica. Yearbook of the Conference of Latin Americanist Geographers 15: 13-22.

Huang, J., R. Wang, F. Li, W. Yang, C. Zhou, J. Jin \& Y. Shi. 2009. Simulation of thermal effects due to different amounts of urban vegetation within the built-up area of Beijing, China. Int. J. Sustainable Dev. World Ecol. 16: 67-76.

Jim, C.Y. \& W. Chen. 2008. Pattern and divergence of tree communities in Taipei's main urban green spaces. Landscape Urban Plann. 84: 312-323.

Jiménez, H. 2010. Programa "Costa Rica: Jardín Botánico de América Tropical" (www.hjimenez.org. Downloaded June 7, 2010).

Kent, M., R.A. Stevens \& L. Zhang. 1999. Urban plant ecology patterns and processes: a case study of the flora of the City of Plymouth, Devon, U.K. J. Biogeogr. 26: 1281-1298. 
Knapp, S., I. Kühn, J. Stolle \& S. Klotz. 2009. Changes in the functional composition of a Central European urban flora over three centuries. Perspectives in Plant Ecology, Evolution and Systematics. In Press (DOI: 10.1016/j.ppees.2009.11.001).

Kong, F. \& N. Nakagoshi. 2005. Spatial-temporal gradient of urban green spaces in Jinan, China. Landscape Urban Plann. 78: 147-164.

Leiva C.A. (comp.). 2004. Costa Rica en fotografías antiguas. Jadine, San José, Costa Rica.

Lücking, R., T. Lumbsch, J. Di Stefano-Gandolfi, D. Lizano, J. Carranza-Velázquez, A. Bernecker-Lücking, J. Chaves-Chaves \& L. Umaña-Tenorio. 2008. Eremithallus costaricensis (Ascomycota: Lichinomycetes: Eremothallales), a new fungal lineage with a novel lichen symbiotic lifestyle discovered in an urban relict forest in Costa Rica. Symbiosis 46: 161-170.

Lundholm, J.T \& A. Marlin. 2006. Habitat origins and microhabitat preferences of urban plant species. Urban Ecosyst. 9:139-159.

McKinney, M. 2008. Effects of urbanization on species richness: A review of plants and animals. Urban Ecosyst. 11:161-176.

McPherson, E., J. Simpson, P. Peper \& E. Aguaron. 2008. Urban Forestry and Climate Change. USDA Forest Service, Albany, California.

Matos, S., C. Santos \& D. Chevalier. 2002. Fire and restoration of the largest urban forest of the world in Rio de Janeiro City, Brazil. Urban Ecosys. 6:151-161.

Maurer, U., T. Peschel \& S. Schmitz. 2000. The flora of selected urban land-use types in Berlin and Postdam with regard to nature conservation in cities. Landscape Urban Plann. 46: 209-215.

Méndez, O. \& L.A. Fournier. 1980. Los líquenes como indicadores de la contaminación atmosférica en el área metropolitana de San José, Costa Rica. Rev. Biol. Trop. 28: 31-39.

Méndez, E. 2005. Flora y vegetación del centro urbano de Luján de Cuyo. Rev. FCA UNCuyo 37: 67-74.

Monge-Nájera, J., M.I. González, M. Rivas \& V.H. Méndez-Estrada. 2002a. Twenty years of lichen cover change in a tropical habitat (Costa Rica) and its relation with air pollution. Rev. Biol. Trop. 50: 309-319.

Monge-Nájera, J., M.I. González, M. Rivas\& V.H. Méndez-Estrada. 2002b. A new method to assess air pollution using lichens as bioindicators. Rev. Biol. Trop. 50: 321-325.

Niggemann, M., J. Jetzkowitz, S. Brunzel, M. Wichmann \& R. Bialozyt. 2009. Distribution patterns of plants explained by human movement behavior. Ecological Model. 220: 1339-1346.

Nishida, K., I. Nakamura \& C.O. Morales. 2009. Plants and butterflies of a small urban preserve in the Central Valley of Costa Rica. Rev. Biol. Trop. 57: 31-67.

Paucharda, A., M. Aguayo, R. Peña \& R. Urrutia. 2006. Multiple effects of urbanization on the biodiversity of developing countries: The case of a fast-growing metropolitan area (Concepción,Chile). Biol. Conser. 127: $272-281$.

Pedlowski, M., J. Corabi \& N. Heynen. 2002. Urban forest and environmental inequality in Campos dos Goytacazes, Rio de Janeiro, Brazil. Urban Ecosyst. 6: 9-20.

Polk, M., K. Young \& K. Crews-Meyer. 2005. Biodiversity conservation implications of landscape change in an urbanizing desert of South western. Urban Ecosyst. 8: $313-334$

Prach, K., P. Pyšek \& M. Bastl. 2001. Spontaneous vegetation succession in human-disturbed habitats: A pattern across seres. Appl. Veg. Sci. 4: 83-88.

Puth, L. \& C. Burns. 2008. New York's nature: a review of the status and trends in species richness across the metropolitan region. Diver. Distr. 15: 12-21.

Pysek, P. 1998. Alien and Native Species in Central European Urban Floras: A Quantitative Comparison. J. Biogeogr. 25: 155-163.

Shaltout, K.H. \& M. El-Sheikh. 2002. Vegetation of the urban habitats in the Nile Delta region, Egypt. Urban Ecosyst. 6: 205-221.

Shaw, S. \& K. Nishida. 2005. A new species of gregarious Meteorus (Hymenoptera: Braconidae) reared from caterpillars of Venadicodia caneti (Lepidoptera: Limacodidae) in Costa Rica. Zootaxa 1028: 49-60.

Smith, R., K. Thompson, J.G. Hodgson \& P. Warren. 2006 Urban domestic gardens (IX): composition and richness of the vascular plant flora, and implications for native biodiversity. Biol. Conser. 129: 312-322.

Vilela, J. 2004. Distribución del arbolado urbano en la ciudad de Fuenlabrada y su contribución a la calidad del aire. Estudios Territoriales 36: 419-427.

Webb, R., D. Boyer \& R. Turner. 2010. Repeat Photography: Methods and Applications in the Natural Sciences. Island, Washington, USA.

Williams, N., M. Schwartz, P. Vesk, M. McCarthy, A. Hahs, S. Clemants, R. Corlett, R. Duncan, B. Norton, K. Norton \& M. McDonnell. 2009. A conceptual framework for predicting the effects of urban environments on flora. J. Ecol. 97: 4-9. 


\section{APPENDIX 1}

Species collected in the late 19th and early 20th century

\begin{tabular}{|c|c|c|}
\hline Species & Date & Collector \\
\hline Acalypha macularis & 1906-10-28 & Otón Jiménez 46 \\
\hline Acalypha amentacea & 1906-12-03 & Otón Jiménez 95 \\
\hline Acanthus mollis & 1931-03-25 & Juvenal Valerio 73 \\
\hline Achimenes grandiflora & $1888-07-31$ & Paul Biolley \\
\hline Achimenes longiflora & 1888-07-31 & Paul Biolley \\
\hline Acmella oppositifolia & $1889-06-20$ & H. Pittier 1091 \\
\hline Acmella radicans & 1892-11-30 & A. Tonduz 7185 \\
\hline Acnistus arborescens & 1893-06-30 & A. Tonduz \\
\hline Adiantum colpodes & 1896-07-31 & A. Tonduz 8064 \\
\hline Adiantum patens & 1888-01-15 & H. Pittier 48 \\
\hline Adiantum concinnum & 1945-08-06 & Richard W. Holm 991 \\
\hline Aeschynomene villosa & $1890-12-31$ & Paul Biolley \\
\hline Agave wercklei & $1907-02-13$ & Anastasio Alfaro \\
\hline Ageratum conyzoides & 1931-01-03 & Fernando Solís 2 \\
\hline Ageratum microcarpum & 1893-01-31 & A. Tonduz 7281 \\
\hline Albizia adinocephala & 1890-07-31 & Paul Biolley \\
\hline Aleurites moluccanus & 1906-10-30 & Otón Jiménez 127 \\
\hline Alternanthera laguroides & 1896-06-30 & A. Tonduz 10135 \\
\hline Amaranthus hybridus & 1888-07-31 & H. Pittier 398 \\
\hline Amphilophium paniculatum & $1888-01-31$ & H. Pittier 975 \\
\hline Anagallis pumila & $1893-02-28$ & A. Tonduz \\
\hline Anemia hirsuta & $1888-07-31$ & Paul Biolley 912 \\
\hline Anoda cristata & 1931-01-03 & Fernando Solís 9 \\
\hline Anredera cordifolia & 1937-10-21 & José Antonio Echeverría 4007 \\
\hline Anthurium acutangulum & 1896-09-30 & A. Tonduz 10360 \\
\hline Aphelandra deppeana & 1896-12-31 & A. Tonduz 10401 \\
\hline Archibaccharis torquis & $1892-11-30$ & A. Tonduz 1535 \\
\hline Archibaccharis schiedeana & $1889-12-12$ & A. Tonduz 1496 \\
\hline Arenaria lanuginosa & $1889-06-20$ & A. Tonduz \\
\hline Arracacia xanthorrhiza & 1904-11-29 & A. Tonduz 17454 \\
\hline Arthrostemma ciliatum & 1888-01-15 & H. Pittier \\
\hline Asclepias curassavica & $1892-07-31$ & A. Tonduz 436 \\
\hline Asplenium pumilum & 1888-07-31 & Paul Biolley 906 \\
\hline Asplenium aethiopicum & 1889-06-20 & A. Tonduz 1095 \\
\hline Asplenium myriophyllum & $1888-07-31$ & Paul Biolley 516 \\
\hline Asplenium formosum & 1888-07-31 & Paul Biolley 914 \\
\hline Aster spinosus & $1888-07-31$ & Paul Biolley 990 \\
\hline Athyrium palmense & $1888-07-31$ & H. Pittier 905 \\
\hline Axonopus compressus & 1890-08-31 & A. Tonduz 2828 \\
\hline Baccharis braunii & 1896-09-30 & A. Tonduz 10882 \\
\hline Begonia cuculata & 1896-12-31 & A. Tonduz 10399 \\
\hline Bidens pilosa & 1888-07-31 & Paul Biolley 980 \\
\hline Blechnum occidentale & $1888-07-31$ & Paul Biolley 921 \\
\hline
\end{tabular}


APPENDIX 1 (Continued)

Species collected in the late 19th and early 20th century

\begin{tabular}{|c|c|c|}
\hline Species & Date & Collector \\
\hline Blechum brownei & 1890-12-07 & A. Tonduz 3204 \\
\hline Blechum pyramidatum & $1931-03-25$ & Juvenal Valerio 79 \\
\hline Bletia purpurea & 1903-05-31 & H. Pittier 16726 \\
\hline Bouvardia glabra & 1896-09-30 & A. Tonduz \\
\hline Brassica campestris & $1889-10-31$ & A. Tonduz 3535 \\
\hline Browallia americana & $1892-11-30$ & A. Tonduz 7183 \\
\hline Brugmansia candida & $1892-07-31$ & A. Tonduz 442 \\
\hline Byttneria aculeata & $1888-07-31$ & Paul Biolley \\
\hline Byttneria carthaginensis & 1888-09-30 & Paul Biolley \\
\hline Calea axillaris & $1889-11-23$ & A. Tonduz 1430 \\
\hline Calea urticifolia & 1931-01-03 & Fernando Solís \\
\hline Calliandra calothyrsus & $1888-08-31$ & H. Pittier \\
\hline Calyptocarpus wendlandii & $1890-05-15$ & A. Tonduz 2334 \\
\hline Calyptocarpus wendlandii & 1931-01-03 & Fernando Solís \\
\hline Calyptranthes pallens & $1929-05-21$ & Manuel Valerio \\
\hline Campyloneurum irregulare & $1888-07-31$ & Paul Biolley 922 \\
\hline Campyloneurum xalapense & 1902-03-31 & Anastasio Alfaro \\
\hline Canavalia villosa & $1889-11-23$ & A. Tonduz \\
\hline Canna indica & 1906-11-30 & 101 \\
\hline Capsicum аппиит & $1895-09-30$ & Paul Biolley \\
\hline Cassia tonduzii & 1896-06-30 & A. Tonduz \\
\hline Cassia patellaria & $1888-10-31$ & H. Pittier \\
\hline Cassia pubescens & $1889-09-30$ & A. Tonduz \\
\hline Casuarina cunninghamiana & 1906-09-29 & Otón Jiménez 84 \\
\hline Cedrela tonduzii & 1903-06-30 & H. Pittier \\
\hline Centaurium quitense & 1890-12-07 & A. Tonduz \\
\hline Centradenia inaequilateralis & 1888-07-31 & Paul Biolley \\
\hline Cestrum aurantiacum & $1888-07-31$ & Paul Biolley \\
\hline Cestrum lanatum & 1893-01-31 & A. Tonduz \\
\hline Cestrum macrophyllum & 1901-02-28 & A. Tonduz \\
\hline Cestrum warscewiczii & 1888-01-15 & H. Pittier 47 \\
\hline Cestrum glanduliferum & 1929-05-05 & A. M. Brenes 37 \\
\hline Cestrum tomentosum & 1941-12-09 & José Antonio Echeverría 230 \\
\hline Cestrum nocturnum & 1936-04-29 & Juvenal Valerio \\
\hline Chamaedorea costaricana & 1905-03-30 & Paul Biolley \\
\hline Chamaesyce lasiocarpa & $1892-02-28$ & A. Tonduz \\
\hline Chenopodium ambrosioides & 1888-01-31 & H. Pittier \\
\hline Chiococca alba & $1890-07-31$ & Paul Biolley \\
\hline Chloris virgata & 1890-09-15 & A. Tonduz 2937 \\
\hline Chromolaena odorata & 1896-08-31 & A. Tonduz 10894 \\
\hline Chrysophyllum cainito & 1902-09-15 & H. Pittier \\
\hline Cinchona pubescens & 1937-09-07 & A. M. Brenes \\
\hline Cissampelos pareira & $1896-06-22$ & A. Tonduz \\
\hline
\end{tabular}


APPENDIX 1 (Continued)

Species collected in the late 19th and early 20th century

\begin{tabular}{|c|c|c|}
\hline Species & Date & Collector \\
\hline Citharexylum donnell-smithii & 1895-01-31 & A. Tonduz 9623 \\
\hline Cleome costaricensis & $1889-05-20$ & A. Tonduz 1086 \\
\hline Cleome pilosa & $1889-11-28$ & A. Tonduz 1450 \\
\hline Clerodendrum thomsonae & 1928-04-29 & Manuel Valerio 64 \\
\hline Clibadium surinamense & $1885-07-31$ & Paul Biolley 976 \\
\hline Cnidoscolus urens & 1903-05-31 & H. Pittier \\
\hline Coelogyne speciosa & $1932-11-11$ & A. M. Brenes 366 \\
\hline Commelina diffusa & $1890-10-31$ & A. Tonduz 3049 \\
\hline Commelina leiocarpa & 1931-01-03 & Fernando Solís 45 \\
\hline Consolida ajacis & $1940-11-20$ & María del Carmen Roviralta 10 \\
\hline Conyza bonariensis & $1892-10-31$ & A. Tonduz 857 \\
\hline Conyza schiedeana & $1892-10-31$ & A. Tonduz 856 \\
\hline Conyza canadensis & 1931-01-03 & Fernando Solís \\
\hline Cordia spinescens & $1896-06-30$ & A. Tonduz 8879 \\
\hline Cordia eriostigma & 1933-04-15 & Fernando Solís 527 \\
\hline Crinum erubescens & $1893-08-31$ & A. Tonduz 8214 \\
\hline Crotalaria rotundifolia & $1892-06-30$ & A. Tonduz \\
\hline Crotalaria vitellina & $1892-09-30$ & A. Tonduz \\
\hline Crotalaria cajanifolia & $1888-03-31$ & Paul Biolley \\
\hline Crotalaria acapulcensis & 1942-09-30 & José Antonio Echeverría \\
\hline Croton hoffmannii & $1896-06-30$ & A. Tonduz \\
\hline Croton draco & $1892-12-31$ & A. Tonduz 7261 \\
\hline Cuphea carthagenensis & $1891-06-30$ & A. Tonduz \\
\hline Cuphea wrightii & $1889-07-27$ & A. Tonduz \\
\hline Cuphea appendiculata & $1888-07-31$ & Paul Biolley \\
\hline Cyclanthera tonduzii & $1889-11-28$ & A. Tonduz 1449 \\
\hline Cyclospermum leptophyllum & $1889-08-12$ & A. Tonduz 1287 \\
\hline Cynoglossum amabile & 1940-11-13 & María del Carmen Roviralta 8 \\
\hline Cyperus papyrus & 1906-09-29 & Otón Jiménez 76 \\
\hline Cyperus flavescens & $1892-10-31$ & A. Tonduz 1531 \\
\hline Cyperus niger & $1890-12-07$ & A. Tonduz \\
\hline Cyperus tenuis & 1892-07-31 & A. Tonduz 433 \\
\hline Cyperus involucratus & 1896-09-30 & A. Tonduz 10888 \\
\hline Cyperus mutisii & 1906-11-11 & Otón Jiménez 69 \\
\hline Cyperus hermaphroditus & 1906-11-11 & Otón Jiménez 68 \\
\hline Cystopteris fragilis & $1888-07-31$ & Paul Biolley 414 \\
\hline Dahlia rosea & $1888-01-15$ & H. Pittier 58 \\
\hline Dalea cliffortiana & $1890-07-31$ & A. Tonduz \\
\hline Delilia biflora & $1896-12-31$ & A. Tonduz 10893 \\
\hline Delilia biflora & 1931-01-03 & Fernando Solís \\
\hline Dennstaedtia distenta & 1922-08-07 & Manuel Valerio \\
\hline Desmodium affine & $1888-07-31$ & Paul Biolley \\
\hline Desmodium distortum & $1902-11-30$ & H. Pittier \\
\hline
\end{tabular}


APPENDIX 1 (Continued)

Species collected in the late 19th and early 20th century

\begin{tabular}{|c|c|c|}
\hline Species & Date & Collector \\
\hline Desmodium intortum & $1892-11-26$ & A. Tonduz \\
\hline Desmodium tortuosum & $1902-12-31$ & H. Pittier \\
\hline Desmodium maxonii & 1931-01-03 & Fernando Solís \\
\hline Dicliptera unguiculata & 1931-01-03 & Fernando Solís 10 \\
\hline Dieffenbachia oerstedii & $1890-05-15$ & A. Tonduz 2558 \\
\hline Dietes grandiflora & 1931-04-11 & A. M. Brenes 89 \\
\hline Dorstenia contrajerva & $1896-07-31$ & A. Tonduz \\
\hline Dracaena fragrans & $1934-02-27$ & Juvenal Valerio 1078 \\
\hline Drymaria cordata & 1889-10-31 & A. Tonduz \\
\hline Drymaria villosa & $1892-06-30$ & A. Tonduz \\
\hline Drymonia serrulata & 1896-07-31 & A. Tonduz \\
\hline Dryopteris opposita & 1888-07-31 & Paul Biolley 413 \\
\hline Dryopteris patula & 1888-07-31 & Paul Biolley 923 \\
\hline Dryopteris litigiosa & 1896-09-30 & A. Tonduz 132088 \\
\hline Duranta erecta & $1934-12-30$ & A. M. Brenes 22868 \\
\hline Echinochloa crus-galli & $1888-12-31$ & H. Pittier 229 \\
\hline Echinochloa crus-pavonis & 1890-09-08 & 3016 \\
\hline Echinocystis coulteri & 1889-11-30 & A. Tonduz \\
\hline Eclipta prostrata & 1892-10-31 & A. Tonduz 9570 \\
\hline Eleocharis elegans & 1906-11-11 & Otón Jiménez 74 \\
\hline Elephantopus mollis & 18-11-1931 & Manuel Quirós 371 \\
\hline Elephantopus scaber & 1889-11-30 & A. Tonduz \\
\hline Elephantopus spicatus & $1889-12-12$ & A. Tonduz 1494 \\
\hline Encyclia livida & $1890-03-10$ & A. Tonduz 2176 \\
\hline Eragrostis ciliaris & 1890-09-15 & A. Tonduz 2935 \\
\hline Eragrostis hypnoides & 1890-05-15 & 2477 \\
\hline Eragrostis mexicana & 1890-09-15 & A. Tonduz 2932 \\
\hline Erechtites hieraciifolius & $1888-08-26$ & H. Pittier 462 \\
\hline Erigeron cuneifolium & $1888-11-30$ & H. Pittier 649 \\
\hline Eryngium carlinae & 1889-06-30 & A. Tonduz 1124 \\
\hline Erythrina berteroana & 1899-12-31 & A. Tonduz \\
\hline Erythrina berteroana & 1934-03-14 & Álvaro Sánchez \\
\hline Erythrina crista-galli & 1931-04-01 & Fernando Solís \\
\hline Eucalyptus globulus & 1889-11-30 & A. Tonduz \\
\hline Eucalyptus globulus & 1941-08-20 & José Antonio Echeverría 25 \\
\hline Euphorbia heterophylla & 1889-10-31 & A. Tonduz \\
\hline Euphorbia heterophylla & 1931-01-03 & Fernando Solís \\
\hline Euphorbia pulcherrima & 1931-03-25 & A. M. Brenes \\
\hline Ficus jimenezii & 1906-11-29 & A. Tonduz 17536 \\
\hline Ficus velutina & 1901-11-30 & H. Pittier \\
\hline Ficus pertusa & 1901-11-30 & H. Pittier \\
\hline Ficus goldmanii & $1897-10-31$ & A. Tonduz \\
\hline Ficus costaricana & $1901-11-30$ & H. Pittier \\
\hline
\end{tabular}


APPENDIX 1 (Continued)

Species collected in the late 19th and early 20th century

\begin{tabular}{|c|c|c|}
\hline Species & Date & Collector \\
\hline Fimbristylis dichotoma & $1893-12-31$ & A. Tonduz 1812 \\
\hline Galeana pratensis & $1889-07-27$ & A. Tonduz 1237 \\
\hline Galeana pratensis & 1931-01-03 & Fernando Solís \\
\hline Galinsoga quadriradiata & $1888-07-31$ & Paul Biolley 994 \\
\hline Galinsoga quadriradiata & $1889-08-12$ & A. Tonduz 253 \\
\hline Gonolobus edulis & $1888-07-31$ & Paul Biolley 938 \\
\hline Gossypium barbadense & $1893-05-31$ & A. Tonduz \\
\hline Graptophyllum pictum & $1931-03-25$ & A. M. Brenes 24416 \\
\hline Grevillea banksii & 1938-01-11 & Jorge León 1003 \\
\hline Habenaria clypeata & 1903-08-31 & H. Pittier 16722 \\
\hline Hamelia patens & 1889-07-31 & A. Tonduz \\
\hline Hauya elegans & 1930-07-08 & Manuel Valerio 51 \\
\hline Heliotropium indicum & $1889-10-31$ & A. Tonduz 1146 \\
\hline Heliotropium procumbens & $1890-05-31$ & A. Tonduz 2430 \\
\hline Heteranthera reniformis & $1888-10-31$ & H. Pittier 553 \\
\hline Heterocentron glandulosum & 1892-11-30 & A. Tonduz 1541 \\
\hline Hibiscus rosa-sinensis & $1896-12-31$ & A. Tonduz \\
\hline Hibiscus rosa-sinensis & $1931-04-23$ & Vitalia Sáenz 96 \\
\hline Holmskioldia sanguinea & 1941-11-10 & José Antonio Echeverría 92 \\
\hline Homalocladium platycladum & 1940-12-07 & José Antonio Echeverría 4015 \\
\hline Hydrocotyle ranunculoides & $1889-12-31$ & A. Tonduz 1688 \\
\hline Hymenocallis littoralis & 1905-06-29 & A. Tonduz \\
\hline Hypericum uliginosum & 1888-07-31 & Paul Biolley \\
\hline Hypoxis decumbens & $1893-06-30$ & A. Tonduz 8028 \\
\hline Hyptis suaveolens & $1889-10-31$ & A. Tonduz \\
\hline Hyptis mutabilis & 1893-01-02 & A. Tonduz \\
\hline Hyptis capitata & 1931-01-03 & Fernando Solís \\
\hline Hyptis pectinata & 1931-01-03 & Fernando Solís \\
\hline Hyptis urticoides & 1931-01-03 & Fernando Solís \\
\hline Indigofera costaricensis & 1892-10-31 & A. Tonduz \\
\hline Indigofera tephrosioides & 1888-07-31 & Paul Biolley \\
\hline Inga densiflora & 1934-05-19 & Collector data missing \\
\hline Inga vera & $1888-07-31$ & H. Pittier \\
\hline Inga leptoloba & 1888-05-31 & H. Pittier \\
\hline Inga oerstediana & $1888-05-31$ & H. Pittier \\
\hline Ipomoea alba & 1932-01-06 & Fernando Solís 405 \\
\hline Ipomoea aristolochiifolia & 1933-01-01 & Manuel Quirós 608 \\
\hline Ipomoea batatas & 1932-01-06 & Fernando Solís 407 \\
\hline Ipomoea carnea & 1931-03-25 & Juvenal Valerio 81 \\
\hline Ipomoea parasitica & 1931-01-04 & Fernando Solís 91 \\
\hline Iresine diffusa & 1893-01-01 & A. Tonduz 7241 \\
\hline Jaegeria hirta & 1888-0-731 & Paul Biolley 940 \\
\hline Jaltomata repandidentata & 1928-10-05 & A. M. Brenes \\
\hline
\end{tabular}


APPENDIX 1 (Continued)

Species collected in the late 19th and early 20th century

\begin{tabular}{|c|c|c|}
\hline Species & Date & Collector \\
\hline Jasminum revolutum & 1896-09-30 & A. Tonduz \\
\hline Jatropha gossypiifolia & 1906-12-03 & Otón Jiménez 94 \\
\hline Justicia crenata & $1887-05-31$ & Paul Biolley 1013 \\
\hline Justicia aurea & 1940-11-13 & María del Carmen Roviralta 9 \\
\hline Koellikeria erinoides & $1888-07-31$ & Paul Biolley \\
\hline Kyllinga odorata & $1892-07-31$ & A. Tonduz 434 \\
\hline Lagerstroemia indica & 1891-03-31 & H. Pittier \\
\hline Lantana urticifolia & $1889-08-31$ & A. Tonduz \\
\hline Lantana hirta & 1888-07-31 & A. Tonduz \\
\hline Laportea aestuans & 1936-11-14 & Juvenal Valerio 1388 \\
\hline Lasiacis sorghoidea & 1893-01-01 & A. Tonduz 7234 \\
\hline Lasiacis swartziana & $1888-02-20$ & H. Pittier 81 \\
\hline Lasiacis sorghoidea & 1931-01-03 & Fernando Solís \\
\hline Lastreopsis effusa & $1889-07-27$ & A. Tonduz 1128 \\
\hline Leersia hexandra & $1889-10-31$ & A. Tonduz 1383 \\
\hline Leonurus japonicus & 1890-09-30 & A. Tonduz 1399 \\
\hline Leonurus japonicus & 1931-01-03 & Fernando Solís 25 \\
\hline Lepidium bipinnatifidum & $1892-07-31$ & A. Tonduz 437 \\
\hline Ligustrum vulgare & 1931-04-14 & Fernando Solís 192 \\
\hline Limonium sinuatum & 1931-04-11 & A. M. Brenes 87 \\
\hline Lindernia diffusa & $1893-02-28$ & A. Tonduz \\
\hline Lipocarpha micrantha & 1941-11-06 & José Antonio Echeverría 133 \\
\hline Lippia myriocephala & $1892-11-26$ & Paul Biolley \\
\hline Lippia alba & 1888-07-31 & Paul Biolley \\
\hline Lippia cardiostegia & $1888-12-31$ & H. Pittier \\
\hline Lobelia xalapensis & $1892-10-31$ & A. Tonduz \\
\hline Loeselia glandulosa & 1931-01-04 & Fernando Solís 80 \\
\hline Lophospermum erubescens & 1930-06-10 & Vitalia Sáenz \\
\hline Ludwigia octovalvis & 1889-10-31 & A. Tonduz \\
\hline Ludwigia peruviana & $1889-10-31$ & A. Tonduz \\
\hline Ludwigia peruviana & $1888-01-15$ & H. Pittier 41 \\
\hline Ludwigia octovalvis & 1931-01-03 & Fernando Solís 19 \\
\hline Malachra radiata & 1889-11-30 & A. Tonduz \\
\hline Malachra alceifolia & 1928-10-05 & A. M. Brenes 19 \\
\hline Malpighia glabra & $1890-04-30$ & A. Tonduz \\
\hline Malpighia mexicana & 1891-08-31 & H. Pittier \\
\hline Malva parviflora & 1891-08-31 & H. Pittier \\
\hline Malvaviscus arboreus & $1892-12-31$ & A. Tonduz \\
\hline Mangifera indica & $1888-05-31$ & H. Pittier 388 \\
\hline Manihot glaziovii & 1890-11-30 & A. Tonduz \\
\hline Marsypianthes chamaedrys & $1892-07-31$ & A. Tonduz \\
\hline Marsypianthes chamaedrys & $1931-01-03$ & Fernando Solís \\
\hline Masdevallia ecaudata & $1890-11-30$ & Paul Biolley 3127 \\
\hline
\end{tabular}


APPENDIX 1 (Continued)

Species collected in the late 19th and early 20th century

\begin{tabular}{|c|c|c|}
\hline Species & Date & Collector \\
\hline Maurandia barclayana & 1928-04-29 & Manuel Valerio \\
\hline Mauria heterophylla & 1894-01-31 & Paul Biolley 8475 \\
\hline Mazus pumilus & 1937-04-11 & Jorge León \\
\hline Mecardonia procumbens & 1892-07-31 & A. Tonduz 7113 \\
\hline Melampodium divaricatum & $1892-07-31$ & A. Tonduz 6960 \\
\hline Melampodium gracile & 1931-01-03 & Fernando Solís \\
\hline Melanthera nivea & 1893-11-30 & A. Tonduz 1558 \\
\hline Melanthera nivea & 1931-01-03 & Fernando Solís \\
\hline Mikania micrantha & 1931-01-03 & Fernando Solís \\
\hline Mildella intramarginalis & $1892-11-26$ & A. Tonduz 7220 \\
\hline Mimosa diplotricha & $1893-02-28$ & A. Tonduz \\
\hline Mimosa pudica & 1888-07-31 & Paul Biolley \\
\hline Mimosa sensitiva & 1889-09-28 & A. Tonduz 1279 \\
\hline Mirabilis jalapa & 1931-01-03 & Fernando Solís 27 \\
\hline Mitracarpus hirtus & 1931-01-03 & Fernando Solís 36 \\
\hline Mollugo verticillata & 1941-03-06 & José Antonio Echeverría 94 \\
\hline Monstera adansonii & $1890-07-31$ & Paul Biolley 2846 \\
\hline Montanoa guatemalensis & $1893-02-28$ & A. Tonduz 7331 \\
\hline Moringa oleifera & 1896-07-31 & Paul Biolley \\
\hline Moritzia lindenii & 1896-09-30 & A. Tonduz 10886 \\
\hline Muhlenbergia tenella & 1890-10-09 & A. Tonduz 3015 \\
\hline Muhlenbergia tenella & 1906-10-30 & Otón Jiménez 50 \\
\hline Myriophyllum aquaticum & 1941-10-18 & Vitalia Sáenz 52 \\
\hline Myrtus communis & 1928-04-29 & Manuel Valerio \\
\hline Nasturtium mexicanum & 1891-08-31 & H. Pittier 6906 \\
\hline Nectandra martinicensis & $1896-06-22$ & A. Tonduz 10104 \\
\hline Nectandra turbacensis & $1897-06-24$ & H. Pittier \\
\hline Nephrolepis occidentalis & 1908-10-06 & Humberto Bertolini \\
\hline Nephrolepis undulata & 1922-08-07 & Manuel Valerio \\
\hline Nicotiana tabacum & $1905-06-29$ & A. Tonduz \\
\hline Ochroma pyramidale & 1941-08-20 & José Antonio Echeverría 27 \\
\hline Oenothera biennis & 1905-06-29 & A. Tonduz 17490 \\
\hline Oenothera rosea & 1936-04-29 & Juvenal Valerio 1119 \\
\hline Oleandra costaricensis & 1908-09-04 & Humberto Bertolini \\
\hline Ophioglossum reticulatum & 1888-07-31 & Paul Biolley 925 \\
\hline Oplismenus burmannii & $1890-12-07$ & A. Tonduz 3124 \\
\hline Oplismenus burmannii & $1906-11-27$ & Otón Jiménez 9 \\
\hline Ornithocephalus bicornis & $1904-12-30$ & Paul Biolley 17512 \\
\hline Oxalis latifolia & $1896-07-31$ & A. Tonduz \\
\hline Pachira aquatica & 1933-04-15 & Fernando Solís 526 \\
\hline Pachyrhizus erosus & 1905-05-30 & A. Tonduz \\
\hline Paspalum costaricense & 1890-10-31 & A. Tonduz 3028 \\
\hline Paspalum saccharoides & 1891-08-31 & H. Pittier 6907 \\
\hline
\end{tabular}


APPENDIX 1 (Continued)

Species collected in the late 19th and early 20th century

\begin{tabular}{|c|c|c|}
\hline Species & Date & Collector \\
\hline Paspalum orbiculatum & 1889-06-30 & H. Pittier 1183 \\
\hline Paspalum distichum & $1888-07-20$ & H. Pittier 306 \\
\hline Paspalum notatum & 1906-11-29 & Otón Jiménez 29 \\
\hline Paspalum conjugatum & 1892-09-30 & A. Tonduz 758 \\
\hline Passiflora adenopoda & $1929-08-30$ & José María Orozco 42 \\
\hline Passiflora apetala & $1896-02-28$ & John Donnell Smith \\
\hline Passiflora edulis & $1905-04-29$ & A. Tonduz \\
\hline Paullinia barbadensis & 1893-01-31 & A. Tonduz \\
\hline Pecluma plumula & 1888-07-31 & Paul Biolley 920 \\
\hline Pedilanthus tithymaloides & 1933-04-06 & Manuel Quirós \\
\hline Pellaea ovata & 1908-10-06 & Humberto Bertolini \\
\hline Peperomia tetraphylla & 1891-11-30 & A. Tonduz 3198 \\
\hline Peperomia lanceolatopeltata & 1892-10-05 & A. Tonduz 7262 \\
\hline Peperomia cooperi & $1888-07-31$ & Paul Biolley 524 \\
\hline Peperomia lignescens & 1898-09-30 & A. Tonduz \\
\hline Peperomia galioides & $1892-11-26$ & A. Tonduz \\
\hline Peperomia angularis & 1893-01-01 & A. Tonduz \\
\hline Peperomia deppeana & 1890-07-31 & A. Tonduz \\
\hline Phaseolus lunatus & 1893-01-02 & A. Tonduz \\
\hline Phaseolus lunatus & 1888-01-15 & H. Pittier 52 \\
\hline Phaseolus lunatus & $1932-12-25$ & Fernando Solís \\
\hline Philadelphus myrtoides & $1889-12-31$ & A. Tonduz 1492 \\
\hline Phlebodium pseudoaureum & $1888-07-31$ & Paul Biolley 900 \\
\hline Phthirusa pyrifolia & $1892-08-31$ & A. Tonduz \\
\hline Phthirusa pyrifolia & 1904-10-30 & A. Tonduz \\
\hline Phyla scaberrima & 1889-07-27 & A. Tonduz \\
\hline Phyllanthus niruri & $1888-07-20$ & H. Pittier \\
\hline Piper bredemeyeri & $1889-06-20$ & H. Pittier 1088 \\
\hline Piper hispidum & 1896-07-31 & A. Tonduz 10154 \\
\hline Piper aduncum & 1896-07-31 & A. Tonduz \\
\hline Piper umbellatum & 1892-06-30 & A. Tonduz 693 \\
\hline Pittiera longipedunculata & $1890-07-12$ & A. Tonduz 3200 \\
\hline Plantago australis & 1894-07-31 & A. Tonduz \\
\hline Plantago major & 1894-07-31 & A. Tonduz \\
\hline Plectranthus amboinicus & 1942-04-29 & Manuel Quirós \\
\hline Pleopeltis macrocarpa & $1889-07-27$ & A. Tonduz 1229 \\
\hline Pleopeltis astrolepis & $1892-07-31$ & A. Tonduz 7121 \\
\hline Pleurothallis listerophora & $1890-07-31$ & Paul Biolley 2986 \\
\hline Pоа аппиа & $1888-05-31$ & H. Pittier 230 \\
\hline Polyclathra cucumerina & 1890-07-12 & A. Tonduz 3200 \\
\hline Polygala platycarpa & $1888-05-31$ & Paul Biolley \\
\hline Polygala costaricensis & $1889-11-28$ & A. Tonduz 1450 \\
\hline Polygala violacea & $1888-07-26$ & Paul Biolley 348 \\
\hline
\end{tabular}


APPENDIX 1 (Continued)

Species collected in the late 19th and early 20th century

\begin{tabular}{|c|c|c|}
\hline Species & Date & Collector \\
\hline Polygonum punctatum & $1892-07-31$ & A. Tonduz \\
\hline Polypodium triseriale & 1888-07-31 & Paul Biolley 899 \\
\hline Polypodium polypodioides & 1905-10-01 & Otón Jiménez 291 \\
\hline Polypodium triseriale & 1888-07-31 & Paul Biolley 417 \\
\hline Ponthieva racemosa & $1890-12-31$ & Paul Biolley 3240 \\
\hline Portulaca oleracea & $1890-03-31$ & H. Pittier \\
\hline Prockia crucis & 1891-05-31 & A. Tonduz \\
\hline Pseuderanthemum cuspidatum & 1888-07-31 & Paul Biolley 942 \\
\hline Psidium solisii & $1932-12-25$ & Fernando Solís 509 \\
\hline Psidium cattleianum & 1909-09-21 & A. Tonduz \\
\hline Psidium cattleianum & 1931-06-07 & Manuel Quirós \\
\hline Psychotria pubescens & 1896-07-31 & A. Tonduz \\
\hline Pterolepis pumila & $1888-12-12$ & H. Pittier 10 \\
\hline Punica granatum & $1905-04-29$ & A. Tonduz 17469 \\
\hline Pycreus rivularis & 1906-10-11 & Otón Jiménez 75 \\
\hline Rhynchelytrum repens & 1906-11-19 & Otón Jiménez 58 \\
\hline Rhynchosia longeracemosa & $1892-11-26$ & A. Tonduz \\
\hline Rhynchospora nervosa & 1906-11-29 & Otón Jiménez 124 \\
\hline Richardia scabra & 1896-07-31 & A. Tonduz \\
\hline Ricinus communis & $1889-11-30$ & A. Tonduz \\
\hline Rivina humilis & 1890-10-31 & A. Tonduz \\
\hline Robinsonella divergens & $1889-11-28$ & A. Tonduz \\
\hline Robinsonella lindeniana & 1931-01-12 & Fernando Solís 110 \\
\hline Rorippa mexicana & $1887-12-20$ & H. Pittier 29 \\
\hline Rosa multiflora & 1892-06-30 & A. Tonduz \\
\hline Rubus urticifolius & $1888-07-31$ & H. Pittier \\
\hline Rumex obtusifolius & 1896-07-31 & A. Tonduz 10123 \\
\hline Russelia sarmentosa & $1889-07-27$ & A. Tonduz \\
\hline Rytidostylis carthaginensis & 1888-07-31 & Paul Biolley \\
\hline Sabazia urticifolia & 1896-07-31 & A. Tonduz 10143 \\
\hline Salvia wagneriana & 1889-11-30 & A. Tonduz 1476 \\
\hline Salvia occidentalis & $1893-01-31$ & A. Tonduz \\
\hline Salvia costaricensis & 1895-10-31 & A. Tonduz \\
\hline Salvia lasiocephala & 1893-01-02 & A. Tonduz \\
\hline Salvia polystachya & $1888-02-20$ & H. Pittier \\
\hline Salvia alvajaca & 1892-07-31 & A. Tonduz 701 \\
\hline Salvia occidentalis & 1931-01-03 & Fernando Solís \\
\hline Salvia officinalis & 1938-03-30 & Manuel Quirós \\
\hline Salvia polystachya & 1931-01-03 & Fernando Solís \\
\hline Salvia tiliifolia & 1931-01-03 & Fernando Solís \\
\hline Salvia wagneriana & 1940-10-19 & María del Carmen Roviralta \\
\hline Sambucus mexicana & 1931-07-07 & Fernando Solís 288 \\
\hline Sambucus mexicana & 1931-07-07 & Fernando Solís 288 \\
\hline
\end{tabular}


APPENDIX 1 (Continued)

Species collected in the late 19th and early 20th century

\begin{tabular}{|c|c|c|}
\hline Species & Date & Collector \\
\hline Sansevieria hyacinthoides & 1941-02-18 & José Antonio Echeverría 4057 \\
\hline Sapium macrocarpum & $1893-07-31$ & A. Tonduz 8209 \\
\hline Scleria hirtella & $1888-11-30$ & H. Pittier 648 \\
\hline Scoparia dulcis & 1889-07-31 & A. Tonduz \\
\hline Scoparia dulcis & 1931-09-04 & Fernando Solís \\
\hline Scutellaria purpurascens & $1891-06-30$ & A. Tonduz \\
\hline Sechium tacaco & $1902-10-31$ & K. Werklé 16674 \\
\hline Selaginella serpens & $1888-07-31$ & Paul Biolley 927 \\
\hline Selaginella cuspidata & $1888-07-31$ & Paul Biolley 929 \\
\hline Senecio hoffmannii & $1888-01-15$ & H. Pittier 39 \\
\hline Senna papillosa & $1896-07-31$ & A. Tonduz \\
\hline Serjania acuta & $1897-03-31$ & A. Tonduz \\
\hline Setaria geniculata & 1890-10-04 & A. Tonduz 3008 \\
\hline Sicyos sertuliferus & $1932-12-25$ & Fernando Solís 1100 \\
\hline Sida haenkeana & $1891-05-31$ & H. Pittier \\
\hline Sigesbeckia jorullensis & 1931-01-03 & Fernando Solís \\
\hline Simarouba glauca & $1944-06-28$ & José Antonio Echeverría 478 \\
\hline Smallanthus maculatus & $1892-07-31$ & A. Tonduz 699 \\
\hline Smilax spinosa & $1889-11-28$ & A. Tonduz 1466 \\
\hline Solanum lanceolatum & 1892-09-30 & A. Tonduz \\
\hline Solanum americanum & $1890-03-25$ & A. Tonduz \\
\hline Solanum americanum & 1931-01-03 & Fernando Solís 33 \\
\hline Solanum umbellatum & 1934-03-17 & Estrella U. de Pacheco \\
\hline Solanum wendlandii & $1932-03-23$ & Collector data missing \\
\hline Sonchus oleraceus & $1890-11-30$ & A. Tonduz 3069 \\
\hline Sorghum bicolor & $1888-07-31$ & H. Pittier 383 \\
\hline Spananthe paniculata & 1896-09-30 & A. Tonduz 10883 \\
\hline Spananthe paniculata & 1931-01-03 & Fernando Solís 64 \\
\hline Spathodea campanulata & 1901-11-30 & H. Pittier 16212 \\
\hline Spermacoce assurgens & $1896-07-31$ & A. Tonduz \\
\hline Spigelia splendens & 1888-07-31 & Paul Biolley \\
\hline Sporobolus indicus & $1890-05-15$ & A. Tonduz 2335 \\
\hline Stachys costaricensis & $1887-12-20$ & H. Pittier 34 \\
\hline Stachys costaricensis & $1896-07-31$ & A. Tonduz \\
\hline Stelis tricuspis & $1888-07-31$ & Paul Biolley 949 \\
\hline Stellaria prostrata & $1890-10-31$ & A. Tonduz \\
\hline Stellaria ovata & 1931-01-03 & Fernando Solís 34 \\
\hline Stemodia verticillata & $1895-01-31$ & A. Tonduz 9621 \\
\hline Struthanthus orbicularis & 1893-01-31 & A. Tonduz \\
\hline Stylosanthes guyanensis & 1896-11-30 & A. Tonduz \\
\hline Synadenium grantii & 1941-11-10 & José Antonio Echeverría 103 \\
\hline Syzygium jambos & $1889-10-31$ & A. Tonduz \\
\hline Tabebuia rosea & $1890-02-28$ & Paul Biolley 2215 \\
\hline
\end{tabular}


APPENDIX 1 (Continued)

Species collected in the late 19th and early 20th century

\begin{tabular}{|c|c|c|}
\hline Species & Date & Collector \\
\hline Tagetes microglossa & 1889-11-28 & A. Tonduz 1451 \\
\hline Talinum paniculatum & $1889-07-27$ & A. Tonduz \\
\hline Tecoma stans & $1889-12-12$ & A. Tonduz 1495 \\
\hline Tetrapterys schiedeana & 1898-07-31 & Paul Biolley \\
\hline Thalictrum lankesteri & $1888-07-31$ & Paul Biolley \\
\hline Thelypteris resinifera & $1886-01-31$ & Paul Biolley 1067 \\
\hline Thunbergia alata & $1936-04-29$ & Juvenal Valerio 1136 \\
\hline Thunbergia erecta & 1940-11-13 & María del Carmen Roviralta 5 \\
\hline Tibouchina longifolia & $1893-12-31$ & A. Tonduz \\
\hline Tinantia erecta & $1888-07-31$ & H. Pittier 391 \\
\hline Tithonia longiradiata & $1890-12-03$ & A. Tonduz 3136 \\
\hline Tournefortia glabra & $1889-06-20$ & A. Tonduz 1089 \\
\hline Tragia volubilis & 1892-07-31 & A. Tonduz \\
\hline Trichilia havanensis & 1941-01-01 & José Antonio Echeverría 4023 \\
\hline Trichilia martiana & $1896-06-22$ & A. Tonduz \\
\hline Trifolium amabile & 1889-08-12 & A. Tonduz \\
\hline Tripogandra purpurascens & $1889-07-27$ & A. Tonduz 1252 \\
\hline Tripogandra serrulata & 1889-06-20 & A. Tonduz 1186 \\
\hline Tripsacum andersonii & 1901-08-31 & Brade 16174 \\
\hline Verbena litoralis & 1936-08-19 & Rafael Roig 9 \\
\hline Vigna adenantha & 1931-01-03 & Fernando Solís \\
\hline Vismia baccifera & 1896-06-30 & A. Tonduz \\
\hline Xylosma flexuosa & $1895-01-31$ & A. Tonduz \\
\hline Xylosma velutina & 1902-03-31 & Paul Biolley \\
\hline Zephyranthes carinata & 1893-06-30 & A. Tonduz 8045 \\
\hline Zexmenia longipes & 1890-12-03 & A. Tonduz 3135 \\
\hline Zexmenia costaricensis & $1892-07-31$ & A. Tonduz 7122 \\
\hline Zexmenia frutescens & $1909-12-30$ & A. Tonduz 17977 \\
\hline
\end{tabular}

